\title{
Dural arterio-venous malformations involving the cavernous sinus: a case report
}

\author{
JOHN A. COSTIN, MEREDiTH A. WEINSTEIN, A. J. BERLIN, \\ RUSSELL W. HARDY, AND F. A. GUTMAN \\ From the Department of Ophthalmology, Cleveland Clinic Foundation, Cleveland, Ohio, USA
}

SUMMARY A case of dural arteriovenous malformation involving the cavernous sinus is reported. The patient was successfully treated with selective embolisation of the fistula. These patients constitute a distinct neuro-ophthalmological syndrome which may be very difficult to diagnose clinically because of the subtle signs and symptoms they present with. Angiography is necessary to make the diagnosis and to differentiate these patients from those with the more common carotidcavernous fistulae.

Percutaneous transfemoral catheter embolisations of arteriovenous malformations of the brain have been reported (Kricheff et al., 1972; Luessenhop et al., 1965; Mahalley and Boone, 1974; Pugatch and Wolpert, 1975). Neurological deficits may result if the embolus lodges in a normal artery. Dural arteriovenous malformations supplied by the external carotid artery are ideally treated by embolisation because the branch supplying the fistula can be selectively catheterised, a procedure that avoids the risk of cerebral ischemia.

It is the purpose of this paper to report on a patient with a dural arteriovenous malformation involving the cavernous sinus and to describe the therapeutic management.

The classification of vascular malformations of the central nervous system will be reviewed with specific attention to anatomy, pathophysiology, and clinical characteristics. Special emphasis will be placed on arteriovenous malformations involving the cavernous sinus, especially dural malformations.

\section{Case report}

A 30-year-old white woman presented with a 5-year history of progressive exophthalmos. The onset was insidious, with no antecedent trauma. The only associated symptoms included a pressure sensation behind the right globe, which was accentuated by stooping or straining, and 3 episodes of 'blurred vision'. The blurring was bilateral and had a left

Address for reprints: Dr John A. Costin, Department of Ophthalmology, Cleveland Clinic, 9500 Euclid Avenue, Cleveland, Ohio 44106, USA homonymous quality. The subjective visual symptoms lasted half an hour and were followed by a headache. A 5-year history of a 'rushing sound' in her right ear was elicited.

Previous investigations for these symptoms had included normal skull and orbital $x$-rays and a negative brain scan.

Ophthalmological examination at the Cleveland Clinic revealed an uncorrected visual acuity of $20 / 15$ in both eyes. External examination showed mild proptosis of the right eye, with Hertel exophthalmometry readings of $20 \mathrm{~mm}$ OD and $16 \mathrm{~mm}$ OS.

Dilated episcleral blood vessels without observable pulsations were seen on slit-lamp examination. A systolic bruit was heard over the right cheek, temple, and globe. The remainder of the examination, including ophthalmoscopy and Goldmann perimetry was normal.

At this point the presumptive diagnosis was an arteriovenous malformation, most likely a carotidcavernous sinus fistula. The patient was admitted to the hospital for angiographic evaluation.

Cerebral angiography was carried out by means of a right femoral catheterisation. The left internal and external carotid arteries, both vertebrals, and the right internal carotid were normal. The right external carotid angiogram showed a dilated middle meningeal artery which fed into a fistula at the level of the foramen spinosum (Figs. 1 and 2). From the foramen spinosum a dilated vein could be traced to the cavernous sinus, where retrograde flow could be demonstrated into dilated ophthalmic veins and thence into a dilated facial vein. The diagnosis of a dural arteriovenous malformation involving the cavernous sinus was established. It was felt that this 


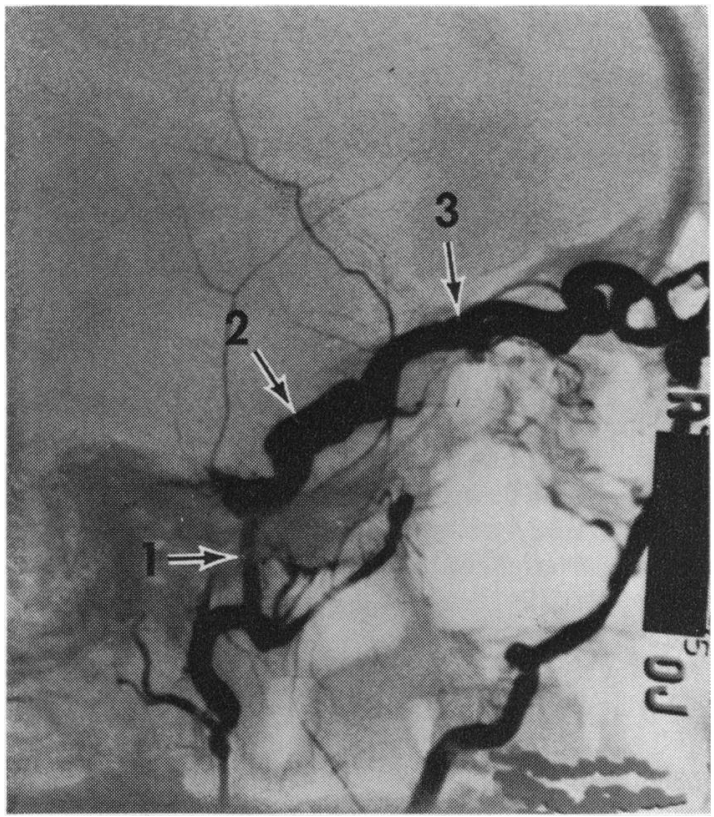

Fig. 1 Lateral view. Selective right external carotid angiogram. The middle meningeal artery (arrow 1) is markedly dilated from the internal maxillary artery to the region of the foramen spinosum (arrow 2), the superior ophthalmic vein (arrow 3), and the facial vein. The middle meningeal and superficial temporal arteries fill poorly

malformation could best be treated by embolisation. By means of a transfemoral artery approach a catheter was selectively threaded into the right external carotid artery. Ground-up pieces of gelfoam were then injected in an attempt to embolise the malformation. Immediately after the injection an angiogram showed complete occlusion of the arteriovenous malformation, with the remainder of the vessels normal (Fig. 3). The patient had no complaints or complications during the procedure, and immediately after the embolisation she stated that the 'rushing sound' in her right ear had disappeared. Her exophthalmos promptly resolved within an hour.

The patient is completely asymptomatic after a year. She has no detectable sequelae from the operation.

\section{Discussion}

Vascular malformations of the central nervous system have been known for at least 300 years. Despite this there is still confusion about their classification and aetiology. A classification which is based on microscopic features has been proposed by Russell and Rubenstein (1971) as follows:

(1) Capillary telangiectasis; (2) cavernous angiomas;

(3) venous malformations; (4) arteriovenous malformations.

Capillary telangiectasias are relatively common lesions that are generally incidental findings at necropsy. They are typically small malformations which generally cause no symptoms. The pons is the commonest location for them, but they can also appear in the cerebral cortex and the white matter.

Cavernous angiomas are uncommon lesions but

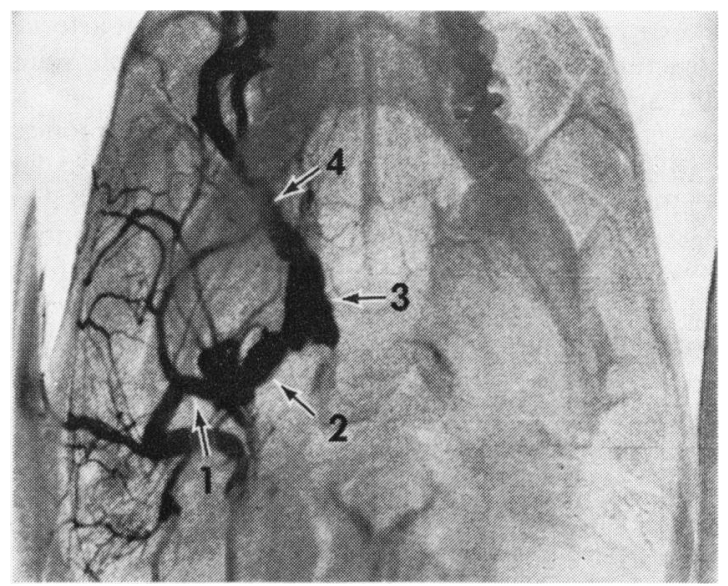

Fig. 2 Base view. The proximal portion of the middle meningeal vessel is dilated (arrow 1). A fistula communication is in the region of the foramen spinosum. $A$ dilated vein (arrow 2) extends from the region of the foramen spinosum to the posterior portion of the cavernous sinus (arrow 3). The superior ophthalmic vein (arrow 4) fills in a retrograde direction

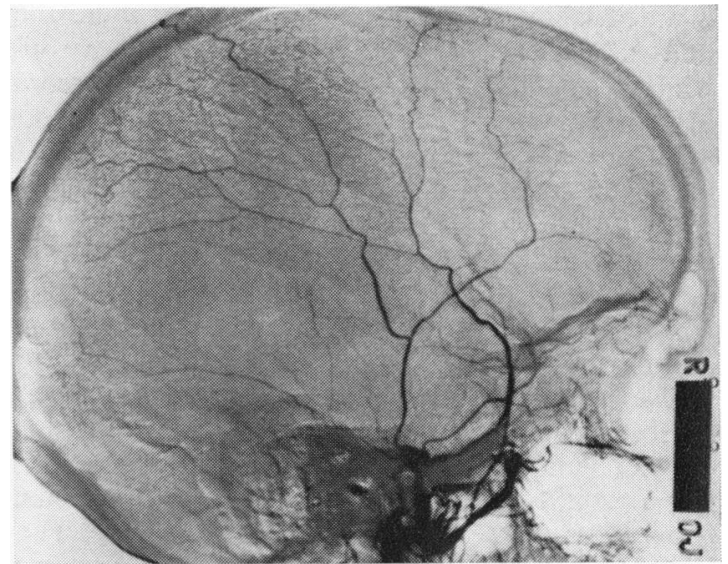

Fig. 3 After embolisation the middle meningeal and superficial temporal arteries fill normally. The fistula communication to the cavernous sinus has been occluded 
extremely important clinically. They are a potential source of bleeding and occasionally rupture, leading to severe intracranial haemorrhage. Moreover, their presence is frequently associated with convulsions. One of the most important facets of these lesions is good access to surgical removal, since they are generally solitary, well defined, and most often located on the surface of the cerebrum.

Venous malformations are most commonly found in the spinal cord and meninges, but are also often found in the scalp and may occur in the orbit. In the last situation they may cause intermittent exophthalmos. Histologically they are very much like arteriovenous malformations except that arterial structures are absent. They consist of multiple veins of varying calibre.

Arteriovenous malformations are the commonest and best described of all the cerebral vascular malformations. William Hunter is credited with first describing patients with abnormal arteriovenous communications in the eighteenth century. Cushing and Baily (1928), as well as Dandy and Follis (1941), published classical papers on a series of cases of arteriovenous 'aneurysms' of the brain. They described the gross appearance of these lesions in addition to the signs and symptoms produced by them.

Pathologically these lesions show great variation. Grossly, they may appear as a tortuous mass or a 'bag of worms'. They are composed of arteries and veins of abnormal calibre and length. The histology is often complex, with specimens varying from well differentiated arteries and veins to thin-walled hyalinised vessels (Newton and Potts, 1974a).

Intracranial arteriovenous malformations are generally classified according to their arterial supply. Under this classification there are 3 types. The first is pure pial arteriovenous malformations, indicating that the arterial supply comes from the vessels which feed the brain substance, that is, the cerebral or cerebellar arteries. Pure dural arteriovenous malformation indicates that the arterial source is from the meningeal vessels which feed the dura mater, that is, branches of the internal carotid artery, external carotid artery, or vertebral artery. Mixed pial dural arteriovenous malformations are those which derive arterial blood from both pial vessels and dural vessels.

The malformations, regardless of which type, are most commonly found in the supratentorial region. Newton and Cronqvist (1969) and McCromick (1966) studied 129 intracranial arteriovenous malformations where angiograms were available. They reported that $80 \%$ of these lesions were supratententorial and $20 \%$ were infratentorial. Of the supratentorial lesions only $6 \%$ could be classified as purely dural, whereas $35 \%$ of the infratentorial lesions fell into this category.

The cavernous sinus is a plexiform sinus composed of arterial and venous channels and neural and connective tissue. It is located extradurally in the lateral sellar space and derives its blood supply from a number of sources. The internal carotid artery supplies numerous dural branches. The external carotid artery via the internal maxillary, ascending pharyngeal, middle meningeal, and accessory meningeal also contributes dural branches (Parkinson, 1972).

Although uncommon, several hundred cases of carotid-cavernous fistulae have been reported since their original description by Travers in 1811. They involve a direct communication between the internal carotid arterial wall and the sinus itself. These fistulae usually are a high-flow and high-pressure system which causes the arterialised blood to flow from the cavernous sinus forward into the ophthalmic veins and orbit. This produces the classical signs and symptoms of carotid-cavernous fistulae: severe ipsilateral headache, accompanying homolateral carotid bruit, progressive exophthalmos, dilated and frequently pulsating episcleral blood vessels, and varying degrees of ophthalmoplegia.

A cavernous sinus fistula or malformation, which is not as well known and which is emerging as a distinct neuro-ophthalmological entity, is one derived from the dural blood vessels in the cavernous sinus. Newton and Hoyt (1970) reviewed the 30 patients with carotid-cavernous fistulae that they had studied from 1962 to 1969 . On retrospective review of the angiograms 11 of these patients were found to have dural arteriovenous malformations involving the cavernous sinus. In 8 of these patients the shunt was fed by meningeal vessels and drained via the cavernous sinus. They found no difference in laterality. The source of the shunt in 3 patients was solely from the external carotid artery. The internal carotid was the sole contributor in 2 , and the remaining 6 cases had shunts fed by both internal and external carotid arteries. Three of their cases were found to be radiographically identical to the case presented here, that is, a middle meningeal artery which fed a fistula situated at the level of the foramen spinosum and drained into the cavernous sinus. All 3 of these cases received their blood supply solely from the external carotid artery.

The signs and symptoms associated with these dural shunts are essentially the same as those seen with the classical carotid cavernous fistulae except they are more subtle and frequently overlooked. The difference in presentation between these 2 entities, that is, dural cavernous fistulae and the classical carotid-cavernous fistulae, is most probably 
secondary to their haemodynamics. The dural malformations are low-flow and low-pressure shunts, while the carotid-cavernous malformations are high-flow and high-pressure shunts. The reason for the difference lies in the anatomy. The dural vessels responsible for these malformations are exceedingly thin walled and small in contradistinction to the internal carotid artery.

These dural arteriovenous shunts may be within the cavernous sinus or directly adjacent to it, or they may involve a more distant sinus and subsequently drain into the cavernous sinus, as in this case (Newton and Potts, 1974b). They may derive their blood supply from the external carotid artery via the middle meningeal, internal maxillary, and ascending pharyngeal arteries or from the internal carotid artery via its dural branches, entering the meningohypophyseal or the artery to the inferior cavernous sinus, or from a combination of both.

The aetiology of these lesions is unknown, but most observers believe they represent a local congenital malformation. The findings of 'mirror' lesions in these patients by Castaigne et al. (1966) supports this theory. They may, however, be due to rupture of the very thin-walled arteries within the cavernous sinus itself, in which case underlying vascular disease could be implicated. Whereas in the past it was believed that $75 \%$ of carotidcavernous fistulae were traumatic and $25 \%$ spontaneous, it may be that the spontaneous fistulae actually represent dural shunts. The definitive diagnosis is made by the angiogram.

Newton and Hoyt's (1970) study reviewed the clinical manifestations in their 11 cases. Their findings have been corroborated by other case reviews (Castaigne et al., 1966; Katsiotis et al., 1944; Kricheff et al., 1972; Mahalley and Boone, 1974; Newton and Hoyt, 1968; Pugatch and Wolpert, 1975; Schlezinger and Schatz, 1973; Wener, et al., 1974).

Headache is one of the most frequent complaints, being severe, unilateral, and often the first symptom. The pain may be localised in the orbital, temple, or forehead region. Propotosis is usually minimal, 2 to $3 \mathrm{~mm}$, but occasionally is moderate. No cases of marked proptosis have been reported thus far. The patient may be unaware of proptosis. Dilated conjunctival vessels are common but may be mild and subtle. The dilated vessels frequently cause the patient to complain of a red eye and may be the initial complaint. There are no reports of pulsatile vessels or pulsatile exophthalmos. Raised introocular pressure is common and generally mild but may be severe.

Unilateral sixth nerve palsy is a frequent finding and may be the first objective sign of the disease.
Its presence correlates well with a shunt in the posterior cavernous sinus. The proposed mechanism for this transient sixth nerve palsy is ischaemia and/or compression of the nerve against the overlying petroclinoid ligament (Newton and Hoyt, 1970).

An objective bruit is found in about $50 \%$ of cases. In Newton and Hoyt's review only 2 of the 11 patients complained of a subjective bruit and both had malformations near the ipsilateral foramen spinosum. Interestingly, our patient had a subjective bruit and a malformation at the ipsilateral foramen spinosum.

The subtle symptoms of a dural arteriovenous malformation create diagnostic problems. Newton and Hoyt's study (1970), as well as our case report, has shown this to be true. They found headaches were most commonly diagnosed as migraine attacks. The 'red eyes' were diagnosed as conjunctivitis, episcleritis, or iritis. The proptosis was most often erroneously attributed to inflammation, infiltrative tumour, or endocrine exophthalmos.

Differentiation of these dural arteriovenous shunts from the more common and less subtle carotid cavernous shunt is important for determination of prognosis and treatment. Because these lesions involve dural vessels and not pial vessels, they may be amenable to embolisation in selected cases. Since the complication of stroke is much less in these dural shunts, selective catheterisation with embolisation of the 'feeding' vessel can be done. This opens a relatively new and quite different modality of treatment for this syndrome. The radiographic diagnosis may be as difficult to make as the clinical one. In order to make a definitive diagnosis of these lesions complete selective cerebral angiography must be performed. Subtraction and magnification techniques are also necessary to isolate the smaller arteries. Common carotid arteriography alone will not suffice in making the diagnosis (Clems and Lodin, 1968; Rosenbaum and Schechter, 1969).

\section{References}

Castaigne, P., Laplane, D., and Djindjian, R. (1966). Communication arterio-veineuse spontanée entre la carotide externe et le sinus caverneux. Revue Neurologique, $114,5-15$.

Clems, F., and Lodin, H. (1968). Non-traumatic external carotid-cavernous sinus fistula. Clinical Radiology, 19, 201-203.

Cushing, H. W., and Bailey. P. (1928). Tumors Arising from the Blood Vessels of the Brain: Angiomatous Malformations and Hemangioblastomas. Charles C Thomas: Springfield, Illinois.

Dandy, W. E., and Follis, R. H. (1941). On the pathology of carotid-cavernous aneurysms (pulsating exophthalmos). American Journal of Ophthalmology, 24, 365-385.

Katsiotis, P., Kiriakopoulos, C., and Taptas, J. (1944). 
Carotid-cavernous sinus fistulae and dural arteriovenous shunts. Vascular Surgery, 8, 60-69.

Kricheff, I. I., Madayag, M., and Braunstein, P. (1972). Transfemoral catheter embolization of cerebral and posterior fossa arteriovenous malformations. Radiology, 103, 107-111.

Luessenhop, A. J., Kachmann, R., Shevlin, W., and Ferrero, A. A. (1965). Clinical evaluation of artificial embolization in the management of large cerebral arteriovenous malformations. Journal of Neurosurgery, 23, 400-417.

Mahalley, S. M., and Boone, S. C. (1974). External carotidcavernous fistula treated by arterial embolization. Journal of Neurosurgery, 40, 110-114.

McCromick, W. F. (1966). The pathology of vascular 'arterio-venous' malformations. Journal of Neurosurgery, 24, 807-816.

Newton, T. H., and Cronqvist, S. (1969). Involvement of dural arteries in intracranial arteriovenous malformations. Radiology, 93, 1071-1078.

Newton, T. H., and Hoyt, W. F. (1968). Spontaneous arteriovenous fistula between dural branches of the internal maxillary artery and the posterior cavernous sinus. Radiology, 91, 1147-1150.

Newton, T. H., and Hoyt, W. F. (1970). Dural arteriovenous shunts in the region of the cavernous sinus. Neuroradiology, 1, 71-81.
Newton, T. H., and Potts, D. G. (1974a). Radiology of the skull and brain. 2, 2522-2528.

Newton, T. H., and Potts, D. G. (1974b). Radiology of the skull and brain, 1 .

Parkinson, D. (1972). Anatomy of the 'cavernous sinus'. Neuro-ophthalmology Symposium of the University of Miami and the Bascom Palmer Eye Institute. Pp. 73-101. C. V. Mosby: St. Louis.

Pugatch, R. D., and Wolpert, S. M. (1975). Transfemoral embolization of an external carotid-cavernous fistula. Journal of Neurosurgery, 42, 94-97.

Rosenbaum, E. A., and Schechter, M. M. (1969). External carotid-cavernous fistulae. Acta Radiologica, 9, 440-444.

Russell, D. S., and Rubenstein, L. J. (1971). Pathology of Tumors of the Nervous System. P. 3. Williams \& Wilkins: Baltimore.

Schlezinger, N. S., and Schatz, N. J. (1973). External carotidcavernous fistula. Transactions of the American Neurological Association, 98, 159-160.

Travers, B. (1811). A case of aneurysm by anastomosis in the orbit, cured by the ligature of the common carotid artery. Medico-Chirurgical Transactions, 2, 1-16.

Wener, L., Chiro, G., and Mendelsohn, R. A. (1974). External carotid-cavernous fistula diagnosed by common carotid arteriography. Journal of Neurosurgery, 41, 494498. 